This is a post-peer-review, pre-copyedit version of an article published in Études théologiques et religieuses. The final authenticated version is available online at https://doi.org/10.3917/etr.934.0529

Published in Études théologiques et religieuses, 2018, no. 93, p. 529-541 which should be cited to refer to this work. DOI: https://doi.org/10.3917/etr.934.0529

\title{
L'enquête de terrain en sciences sociales des religions
}

La démarche ethnographique repose sur un certain nombre de principes méthodologiques. L'enquêteur y adopte une posture d'apprentissage des codes sociaux et s'immerge au sein du milieu social qu'il entend comprendre et étudier. Il pratique l'observation participante tout en prenant en considération la "place» que le collectif lui a progressivement attribuée. Laurent AMIOTTE-SUCHET* montre que, dans l'étude des faits religieux, une telle démarche in situ s'avère particulièrement fructueuse pour comprendre ce qui est réellement en jeu derrière les discours dogmatiques et les pratiques rituelles conventionnelles. Mais cela suppose, pour le chercheur, la mise en ouvre d'un véritable agnosticisme méthodologique questionnant à nouveau les notions mêmes de «pratiques » et de «croyances».

Observateur observé, sujet-objet agissant et agi, le chercheur est, à l'instar de ceux qu'il étudie, un individu collectif dont les représentations évoluent dans leur interaction avec celles des sujetsobjets de son étude ${ }^{1}$.

Aujourd'hui, des formulations telles qu'aller sur le terrain ou être au contact du terrain font partie des stratégies de communication politique. Lors des débats publics, elles sont utilisées par diverses personnalités pour insister sur la proximité qu'elles entretiendraient avec les classes populaires, cherchant par là même à se distinguer d'une classe dirigeante technocratique supposée aveugle aux conditions de vie de celles et ceux dits «d'en bas ». Ces formulations, bien souvent démagogiques, sont ainsi censées insister sur une connaissance approfondie et intime des différents milieux sociaux et, du même coup, sur la capacité du candidat à prendre en considération les réalités empiriques de la vie de celles et ceux qu'il entend soutenir et accompagner. Si les politiques en usent, les chercheurs en sciences humaines et sociales sont également nombreux à se revendiquer d'une approche de terrain, insistant alors sur leur attachement à la prise en compte d'une certaine authenticité. Or, être présent, être témoin d'une situation ou d'un événement ne garantit pas une plus grande objectivité du regard.

En sciences sociales comme en politique, les grands modèles explicatifs macrosociologiques ont montré leurs limites. Théoriser la globalisation du monde ne suffit pas à expliquer la diversité des arrangements locaux, pas plus que les théories de la sécularisation n’ont permis de saisir la pluralisation religieuse contemporaine et ses oscillations. La nécessité de comprendre les mutations sociétales en articulant tendances macrosociologiques globales et arrangements microsociologiques locaux a été au cœur d'une forte revalorisation des approches qualitatives en sciences sociales, et en particulier de la démarche ethnographique, qui déborde maintenant largement les frontières disciplinaires ${ }^{2}$.

Nombre de chercheurs identifient leur démarche comme relevant de l'enquête de terrain. Pourtant, dans de nombreux cas, le qualificatif est abusif car il ne suffit pas d'être présent à un

\footnotetext{
* Laurent AMIOTTE-SUCHET, docteur de l'École pratique des hautes études, est chargé de recherche à la Haute école de santé du canton de Vaud (HESAV - HES-SO) et professeur remplaçant à l'Institut de sciences sociales des religions (ISSR) de l'université de Lausanne.

1 Jean-Claude COMBESSIE, «D'une infraction heureuse aux règles de méthode », Cahiers du Brésil contemporain 43-44 (2001), p. 197.

${ }^{2}$ À ce sujet, Stéphane Beaud et Florence Weber souhaitent combattre la division entre sociologie et anthropologie afin de promouvoir une «ethnographie sociologique »: Stéphane BEAUD, Florence WEBER, Guide de l'enquête de terrain, Paris, La Découverte, coll. « Grands Repères », 2003 (1997), p. 290.
} 
certain moment sur le terrain pour qualifier la démarche d' "enquête de terrain ». Cette dernière renvoie à la mise en œuvre d'une méthodologie spécifique et épistémologiquement fondée. Elle suppose la mise en place d'un certain type de relations avec un réseau d'interconnaissance. Elle ne relève donc pas de la simple observation neutre de situations, mais se caractérise d'abord par une immersion longue et approfondie, supposant donc l'implication du chercheur au sein même du milieu qu'il entend étudier.

Nous commencerons par synthétiser les principes qui fondent l'enquête de terrain et l'observation participante qui lui est intimement associée, puis nous insisterons sur les prérequis essentiels à la mise en œuvre d'une telle démarche d'enquête dans l'étude des faits religieux. Dans un troisième temps, nous donnerons un exemple d'analyse issu de nos propres recherches sur des phénomènes religieux contemporains pour insister sur la plus-value de la méthode ethnographique.

\section{LES PRINCIPES DE L'ENQUETE DE TERRAIN}

L'anthropologie, comme science de l'autre, s'est construite sur les cendres de la découverte du Nouveau Monde. Elle a d'abord eu pour mission de mieux comprendre les us et coutumes de « cette multitude innombrable de nations [féroces et idolâtres] que le démon tenait sous son esclavage ${ }^{3} »$. Les carnets de notes et les écrits des explorateurs, tout autant que les témoignages des colonisés, rendent d'ailleurs compte des perceptions archaïsantes que les uns portaient alors sur les autres ${ }^{4}$. Depuis cette époque, qui constitua le contexte d'émergence de la discipline, l'anthropologie a incontestablement effectué un important saut qualitatif. Son histoire l'amena à passer de l'intérêt pour le sauvage à l'intérêt pour l'autre, pour l'ailleurs ou plus généralement pour l'altérité, remettant ainsi en cause le grand partage entre ici et là-bas et l'essentialisme culturel sur lequel s'était fondé l'ethnocentrisme des regards. C'est à partir des travaux de Bronislaw Malinowski que l'anthropologie découvre la nécessité de recueillir des données de première main par une immersion sur le long terme du chercheur au cœur d'une société étrangère à sa propre culture ${ }^{5}$, afin de ne plus porter sur l'ailleurs un regard européanocentré qui a longtemps vu les autres comme les fossiles vivants d'une préhistoire du monde moderne.

\section{Observation et participation, deux dimensions indissociables}

L'enquête de terrain (ou démarche ethnographique) puise donc ses origines dans la confrontation avec une altérité culturelle. L'ethnographe est celui qui apprend en observant et en participant. Et c'est sur l'association de ces deux activités - observation/participation - que repose la démarche. Le chercheur doit en effet se montrer attentif aux détails de la vie quotidienne, être présent lors des grands événements comme lors des temps ordinaires, noter avec précision tout ce qu'il observe autour de lui et questionner sans relâche ses informateurs sur la signification pour eux de tout ce qui se joue au quotidien. S'inscrivant dans une démarche inductive, le chercheur n'investit donc pas le terrain avec des pistes d'analyse ou des hypothèses scientifiques à éprouver. Il se présente plutôt comme un élève ignorant et maladroit qui entend fonder ses connaissances sur

\footnotetext{
${ }^{3}$ Jean-François LAFITAU, Mœurs des sauvages américains comparées aux mœurs des premiers temps, introduction, choix de textes et notes par Edna Hindie Lemay, 2 vol., Paris, Maspéro, coll. « La Découverte », 1983 (1724), p. 39.

${ }^{4}$ À propos de l'ethnocentrisme des explorateurs, voir Tzvetan ToDOROV, La conquête de l'Amérique. La question de l'autre, Paris, Seuil, 1982. Concernant la perception des Amérindiens, voir Nathan WACHTEL, La vision des vaincus. Les Indiens du Pérou devant la conquête espagnole, Paris, Gallimard, 1971.

${ }^{5}$ Bronislaw MalinowsKi, Les Argonautes du Pacifique occidental, Paris, Gallimard, 1963 (1922).
} 
la base de sa propre socialisation au milieu social étudié. L'humilité dont il fait preuve sur le terrain n'est donc pas une simple forme de politesse visant à se faire accepter, elle repose sur un des principes même de la méthode : mettre l'ethnographe en situation d'apprentissage afin que les acteurs, agissant au quotidien par habitus, soient mis en situation de devoir corriger le comportement du chercheur-apprenti et de devoir verbaliser les représentations sur lesquelles sont censées reposer leurs actions.

L'une des plus importantes conséquences d'une telle démarche réside dans le fait que ce sont les interactions corporelles et verbales renvoyant directement à la présence du chercheur sur le terrain qui font sens, et non celles qui se dérouleraient sous les yeux d'un observateur passif et discret. En d'autres termes, plutôt que de se mettre à distance pour prétendre observer le monde tel qu'il est, l'ethnographe prend pleinement sa place aux cœurs des interactions sociales, afin d'expérimenter le monde tel qu'il s'élabore. Il est donc essentiel que le chercheur occupe une place, c'est-à-dire ait un nom, une identité aux yeux des autres. Dans la méthode sociologique classique basée sur des entretiens directifs ou semi-directifs, les interventions du chercheur durant l'entretien sont limitées et faiblement suggestives, de façon à ne ne pas influencer l'interviewer, ceci dans le but de garantir l'objectivité des données récoltées. Dans la démarche ethnographique, la subjectivité du chercheur est au contraire mobilisée dans les interactions. Il importe qu'il fournisse à ses informateurs sa propre vision nécessairement caricaturale de leur monde, afin que ces derniers résistent aux injonctions du chercheur, corrigent son regard sur leur quotidien et complexifient les représentations que le monde extérieur plaque sur eux. Le chercheur a pour objectif d'être contredit, surpris, désorienté. Il cherche à expérimenter un décalage culturel non seulement pour changer de regard sur les autres, mais également pour parvenir à comprendre autrement son propre monde (ou du moins l'influence que peut avoir sa propre culture sur son regard). En somme, il convient pour le chercheur d'apprendre progressivement à penser comme les autres, de parvenir à leur rendre la parole, sans réduire leur réalité avec des modèles préétablis potentiellement ethnocentriques. De fait, l'ethnographe se montre réticent face aux modélisations théoriques, il privilégie une méthode d'enquête visant à documenter la pluralité des attitudes des acteurs en situation, en considérant qu'un collectif est par principe dynamique, réflexif (voire autoréflexif) et constamment en changement. Comme l'a rappelé très justement Georges Balandier dans l'ensemble de son œuvre, la « tradition » n'est jamais qu'une variante de ladite «modernité », car il n'y a pas de société sans changement.

Aucune société n'échappe à la nécessité de se produire elle-même, sans répit, sous la contrainte de sa propre imperfection et de l'événement. Ce travail sans fin allie continuités et ruptures. Aussi n'autorise-t-il pas à opposer de façon mécaniste tradition et modernité. La première n'est pas un fardeau de formes mortes qui imposerait son inertie à la seconde, elle est le fournisseur de mémoires où le présent recherche une part de ce qui est utile à son accomplissement ${ }^{6}$.

Considérant la valeur heuristique des micro-situations habituellement négligées par les enquêtes macrosociologiques, l'ethnographe ne recherche donc pas la saturation des données. Il mène son enquête comme un détective, suivant les pistes qui s'ouvrent à lui, en combinant l'observation in situ, les entretiens formels et informels avec les acteurs, la consultation d'archives, la réalisation de photographies, la participation à des événements (quitte à s'y impliquer pleinement), etc. $A$ priori, rien ne lui est inutile. Il compile une grande variété d'informations en prenant toujours soin de contextualiser les conditions de leur production. L'ethnographe est conscient qu'il ne recueille pas des données mais qu'il les produit, puisque sa présence fait pleinement partie de la réalité qu'il observe. Armé de son journal de terrain, il réalise dans le même temps, dans une sorte de circulation

${ }^{6}$ Georges BALANDIER, Conjugaisons, Paris, Fayard, 1997, p. 238. 
intellectuelle ininterrompue, l'enregistrement des données, leur contextualisation, leur pré-analyse et l'effort réflexif concernant l'influence de sa propre place sur le terrain.

La phase de production des données peut être ainsi analysée comme une restructuration incessante de la problématique au contact de celles-ci, et comme un réaménagement permanent du cadre interprétatif au fur et à mesure que les éléments empiriques s'accumulent ${ }^{7}$.

\section{Statuts du chercheur et pacte de bienveillance}

Le fait que l'ethnographe occupe une place sur le terrain est donc essentiel. Son statut aux yeux des autres est une variable dépendante de l'enquête, puisque ce dernier évolue constamment et, par conséquent, influence la production des données. Se faire accepter au sein d'un groupe pour mieux le comprendre n'est pas spécifique à l'ethnographie. On peut retrouver ce type de démarche chez les journalistes d'investigation qui, eux aussi, privilégient l'immersion longue pour améliorer la qualité des informations récoltées. Dans leur démarche, l'objectif consiste à mettre en place avec les acteurs des relations de confiance pour que ces derniers quittent un discours de façade et que l'enquêteur puisse alors avoir accès à un discours sincère et authentique. Mais dans la démarche ethnographique, cette corrélation entre statut du chercheur et authenticité des données n'est pas pertinente. Les observations des premiers jours, alors que le chercheur n'est qu'un étranger, sont tout aussi importantes que celles qui suivront, quand l'ethnographe sera introduit, puis accepté au sein du groupe. C'est la variation des pratiques observées et des discours produits, en lien avec les différents statuts du chercheur, qui fait sens. Si l'immersion sur le long terme est essentielle, ce n'est pas pour parvenir à atteindre l'authenticité d'un monde caché derrière une façade conventionnelle, c'est au contraire pour être en mesure d'observer la pluralité des comportements et des discours, à partir d'un ensemble de situations qui varient en fonction des différents statuts de celui qui observe.

L'aventure ethnographique, qui consiste dans l'expérimentation visuelle et linguistique des différences appelle différentes façons de dire, de lire et d'écrire, une variété de versions, le contraire même de l'univocité ${ }^{8}$.

La mise en place de relations avec les acteurs et leur entretien sur le long terme est une marque de fabrique de l'enquête de terrain. Le chercheur acquiert progressivement une place particulière, ni tout à fait dedans ni tout à fait dehors. Cette position au sein du groupe, généralement ambigüe, offre au chercheur un important niveau d'accès aux informations, du fait même qu'il est considéré comme un allié éventuel. Elle lui offre également la possibilité de produire un discours extérieur (potentiellement critique) sur le groupe sans être remis en cause, puisque son statut d'observateur neutre a été antérieurement négocié avec les personnes pour garantir son indépendance. Mais les liens qu'il tisse avec ses informateurs ne sont pas sans conséquences sur ses marges de manœuvre. Pour approfondir sa compréhension du collectif qu'il observe, le chercheur doit toujours aller un peu plus loin, accepter des renvois d'ascenseur, se montrer bienveillant et compréhensif, faire preuve de tact et de diplomatie, s'impliquer dans l'organisation d'un événement, partager certains aspects de sa vie privée avec les personnes qu'il côtoie, etc. L'expérience ethnographique est d'abord une relation de don et de contre-don. Plus qu'une simple empathie, le chercheur doit

\footnotetext{
${ }^{7}$ Jean-Pierre OLIVIER DE SARDAN, «La politique du terrain », Enquête 1 (1995), p. 95.

${ }^{8}$ François LAPLANTINE, La description ethnographique, Paris, Nathan, 1996, p. 117.
} 
«donner de sa personne », apprendre à «être affecté ${ }^{~} »$ par les drames qui se déroulent autour de lui, se sentir concerné par l'avenir du collectif, prouver à ceux qu'il rencontre qu'il est pourvu de bonnes intentions à leur égard. Or, si ce niveau d'implication ouvre de nombreuses portes, il en ferme d'autres. Le chercheur ne peut plus tout dire, au risque de nuire au collectif ou à certains de ses membres. Généralement confronté à de petites unités humaines (un village, un quartier, une communauté), l'ethnographe ne peut aisément garantir l'anonymisation des données qu'il produit, en particulier quand ses observations mettent au jour des conflits ou des relations concurrentielles entre les personnes qu'il côtoie. En ayant eu pour objectif de se laisser socialiser au sein du groupe, en ayant accepté d'y prendre une place, le chercheur a tissé des relations sociales qui s'apparentent à la signature d'un pacte de bienveillance qu'il lui faudra honorer.

\section{PREREQUIS POUR UNE ETHNOGRAPHIE DES EXPRESSIONS RELIGIEUSES CONTEMPORAINES}

Le volet qui précède a permis de poser les grands principes sur lesquels repose l'enquête de terrain :

- immersion sur le long terme pour produire des données de première main ;

- description contextualisée de la vie collective ;

- participation du chercheur à la vie du groupe afin d'y occuper une place ;

- intérêt pour la pluralité des situations plutôt que pour leur répétitivité ;

- approche réflexive du chercheur concernant son propre rôle dans la production des données.

Si ces principes sont communs à toute enquête de terrain en sciences sociales - approche non spéculative, empirique et réflexive ${ }^{10}-$, les recherches ethnographiques portant sur les faits religieux doivent intégrer deux positionnements épistémologiques essentiels afin que l'observation in situ puisse se révéler véritablement heuristique.

\section{Ne pas croire aux pratiques}

Au milieu du $\mathrm{XX}^{\mathrm{e}}$ siècle, la mise en évidence de ce que Gabriel Le Bras appelait la fin de la civilisation paroissiale fut documentée à partir de données statistiques essentiellement issues des registres paroissiaux ${ }^{11}$. La diminution chiffrée de la pratique religieuse dominicale, celle également des rites de passage, servirent de base aux théories prédisant la fin de la religion. Dans ce type d'approche, les chercheurs partaient du principe qu'il existe nécessairement une corrélation entre niveau de croyance et niveau de pratique. Or c'est justement cette assertion - plus ils pratiquent, plus ils croient et inversement - que nombre de travaux proposant une approche ethnographique des rituels remirent en cause. Il existe en effet une pluralité de modes d'engagement. Le fait de pratiquer de manière régulière et intensive au sein d'une communauté croyante ne renvoie pas automatiquement à un niveau de conviction plus élevé, plus exclusif ou plus radical. Il y a là un véritable biais d'analyse, en partie lié à la définition christocentrée de la religion ${ }^{12}$. Pratiquer régulièrement des rites ou être attaché à certains signes religieux ostensibles peut renvoyer à d'autres raisons (celles d'afficher son appartenance à un collectif, d'affirmer une identité religieuse,

\footnotetext{
${ }^{9}$ Jeanne FAVRET-SAADA, « Être affecté », Gradhiva 8 (1990), p. 3-10.

${ }^{10}$ Voir St. BEAUD, F. WEBER, Guide de l'enquête de terrain, op. cit, p. 10.

${ }^{11}$ Gabriel LE BRAS, Études de sociologie religieuse, t. I : Sociologie de la pratique religieuse dans les campagnes françaises, Paris, PUF, 1955.

${ }^{12}$ Voir Daniel Dubuisson, L'Occident et la religion. Mythes, science et idéologie, Bruxelles, Éditions Complexe, 1998.
} 
de se distinguer d'autres groupes de pratiquants, d'adopter une attitude mimétique ou conventionnelle dans une stratégie d'intégration, etc.) que les seules convictions personnelles. En permettant au chercheur d'entretenir des relations amicales et parfois intimes avec les fidèles, l'approche ethnographique met au jour des attitudes et des discours particulièrement décalés par rapport à ce qui s'exprime en surface. L'enquête de terrain a donc pour effet de recentrer l'analyse sur l'empirie, c'est-à-dire ici sur les pratiques observées, tout en permettant au chercheur de relativiser l'existence d'un lien entre croyance et pratique qui conduit trop souvent les analyses (notamment celles véhiculées par les médias) à voir des convictions religieuses fondamentalistes ou intégristes partout où l'attachement à la rigueur d'une pratique cultuelle se manifeste, se renforce ou se revendique.

\section{Ne pas croire aux croyances}

S'il convient donc de se garder d'imputer des croyances aux pratiquants, il importe également (et sans doute plus encore) de ne pas imputer des croyances aux croyants. Si la formule peut surprendre, elle ne fait que répondre aux principes d'une démarche inductive et empirique. Sur le terrain (tout comme lors d'un entretien), le chercheur n'a pas accès à ce que croient les personnes, il a seulement accès à ce qu'elles disent qu'elles croient et à ce qu'elles en expriment publiquement. L'avertissement fut maintes fois formulé : l'ethnographe ne doit pas «jouer à croire aux “croyances" 13 » en restant attentif au fait qu'il n'a accès qu'à des énonciations, effectuées dans un contexte spécifique et devant des interlocuteurs spécifiques (dont le chercheur fait partie). De fait, l'observateur attentif s'apercevra très vite que le contenu de ces énonciations change en fonction des situations et qu'un même individu peut soutenir différents énoncés de croyance, parfois assez éloignés l'un de l'autre (ou même a priori contradictoires), en fonction de ses interlocuteurs ${ }^{14}$. Comme le formulait très justement Jean Pouillon, «seul l'incroyant croit que le croyant croit ${ }^{15}$ ». Sociologues et anthropologues ont longtemps traité les énoncés de croyances des peuples dits traditionnels (et des populations rurales) comme des certitudes tranquilles, radicalement opposées au raisonnement complexe et critique de la science. Cette opposition entre croyance et raison, qui aura fortement imprégné le «croire occidental ${ }^{16}$ », doit être aujourd'hui déconstruite afin de reconnaître aux individus une capacité à prendre leurs distances avec les énoncés qu'ils défendent et à faire preuve de réflexivité vis-à-vis des credo qu'ils relaient. C'est Le Cru et le su de Jean Pouillon $^{17}$, ou encore le mythe grec de Paul Veyne ${ }^{18}$. Tout comme les possédés marocains savent que les djinns ne prennent pas réellement possession de leurs corps et pourtant croient qu'ils se manifestent bien à travers la possession ${ }^{19}$, les catholiques savent que l'hostie n'est pas le corps du Christ tout en croyant qu'il se manifeste bien dans cette médiation. Nous retrouvons là l'apparent

\footnotetext{
13 Jean-Pierre OLIVIER DE SARDAN, «Jeu de la croyance et "je" ethnologique : exotisme religieux et ethno-égocentrisme », Cahiers d'études africaines 28/3-4 (1988), p. 528.

${ }^{14}$ Pour une illustration de l'influence de la situation sur les énoncés de croyance, voir Laurent AMIOTTE-SUCHET, «Énoncés de croyance en situation. Réflexion à partir des "croyances" d'un pèlerin de Lourdes », in Pierre Gisel, Serge Margel (dir.), Le croire au cour des sociétés et des cultures. Différences et déplacements, Turnhout, Brepols, 2011, p. 147-161.

15 Jean Pouillon, Le Cru et le su, Paris, Seuil, 1993.

${ }^{16}$ Roberte HAMAYON, «L'anthropologue et la dualité paradoxale du “croire occidental” », Théologiques 13/1 2005, p. 15-41.

17 J. POUILlon, Le Cru et le su, op. cit.

${ }^{18}$ Paul VeYne, Les Grecs ont-ils cru à leurs mythes?, Paris, Seuil, 1983.

${ }^{19}$ Voir Bertrand Hell, Possession et chamanisme. Les maîtres du désordre, Paris, Flammarion, 1999.
} 
paradoxe qu'Albert Piette décrit très justement comme la mise en «présence de 1 'absent ${ }^{20}$ ». Commentant la célèbre formule d'Octave Mannoni : «Je sais bien... mais quand même ${ }^{21}$ », Dominique Boullier défend l'idée qu'ici comme ailleurs, aujourd'hui comme hier, l'initiation aux pratiques rituelles comme aux énoncés de croyances commence avec la sortie d'une croyance aveugle $^{22}$. C'est en effet en acceptant l'apparent paradoxe qui énonce en même temps que les dieux sont présents et que ce sont les hommes qui les rendent présents, que l'enfant accède à la croyance, c'est-à-dire accepte en somme de s'engager dans une action dont il n'est pas tout à fait dupe mais à laquelle il demeure attaché car elle fait socialement sens pour lui.

Soucieuses de leur objectivité, les sciences sociales ont formulé depuis déjà bien longtemps le principe de l' «agnosticisme méthodologique». Quel que soit l'objet sur lequel il travaille, le chercheur doit en effet faire preuve d'agnosticisme en ce qui concerne les convictions nécessairement idéologiques des personnes qu'il interroge. En d'autres termes, même si les croyances de ses interlocuteurs sont totalement absurdes pour lui, le chercheur doit mettre de côté ses propres convictions afin que ces dernières n'influencent pas son analyse. Mais le principe énoncé plus haut va beaucoup plus loin. Il ne s'agit plus ici de conseiller aux chercheurs de faire preuve d'empathie en faisant semblant de trouver de l'intérêt à des discours qu'ils jugent au fond d'eux-mêmes totalement in-croyables. Il s'agit surtout pour les chercheurs (et l'exercice demeure beaucoup plus facile à dire qu'à faire) d'arrêter de penser que leurs interlocuteurs croient aveuglément en ce qu'ils énoncent afin d'envisager les croyances pour ce qu'elles sont empiriquement : des actes de langage situés et socialement performatifs.

Nous pervertissons aussi bien les sciences transformées en opinion, que les religions transformées en gnose. D'où ce monstre que l'on invoque lorsque l'on pose la question rituelle « Croyez-vous en Dieu ? », comme s'il s'agissait d'une formule de même farine que «Croyez-vous au réchauffement global ?»[...] Pour parler un peu sérieusement de religion, il faut être agnostique, au sens étymologique de ce mot, c'est-à-dire en se gardant de croire à la croyance ${ }^{23}$.

\section{DES APPORTS DE L'ETHNOGRAPHIE A L'ENQUETE}

La mise en œuvre d'une enquête ethnographique dans l'étude des faits religieux, si l'on s'attache dès le départ à ne croire ni aux pratiques ni aux croyances, permet de resserrer la focale sur les expressions publiques d'un religieux en train de se faire, et d'éviter ainsi certains types de surinterprétation consistant à prêter aux acteurs des intentions qui ne sont pas les leurs ou à les ranger dans des catégories essentialisantes alors même qu'ils passent leur temps à résister aux catégorisations dont ils sont l'objet.

Dans le cadre de mes recherches en socio-anthropologie des religions, j'ai pu constater à plusieurs reprises l'intérêt de l'approche ethnographique pour renverser les stéréotypes et éviter le piège de la «croyance ». Les enquêtes que j'ai menées en milieu pentecôtiste dans l'Est de la France (1998-2006) ${ }^{24}$ avaient pour objectif de mieux comprendre les pratiques charismatiques de

\footnotetext{
${ }^{20}$ Albert PIETTE, La religion de près. L'activité religieuse en train de se faire, Paris, Métailié, 1999, p. 254.

${ }^{21}$ Octave MANNONI, «Je sais bien... mais quand même », in Clefs pour l'imaginaire : ou l'Autre Scène, Paris, Seuil, 1969, p. 9-33.

${ }^{22}$ Dominique Boullier, «Au-delà de la croyance : "Je sais bien mais quand même" », Cosmopolitiques 6 (2004), p. $27-47$.

${ }^{23}$ Bruno LATOUR, « Science et Raison : une comédie des erreurs », Cosmopolitiques 6 (2004), p. 51.

${ }^{24}$ Laurent AMIOTTE-SUCHET, Pratiques pentecôtistes et dévotion mariale : analyse comparée des modes de mise en présence du divin, Thèse de doctorat en sociologie des religions, École pratique des hautes études, Section des sciences religieuses (sous la direction de Jean-Paul Willaime), 2006.
} 
ces Églises de convertis ${ }^{25}$. La tentation était grande de plaquer sur ces communautés les analyses durkheimiennes de l'effervescence des profondeurs ${ }^{26}$, en opposant un protestantisme réformé intellectuel et réflexif à un « protestantisme émotionnel ${ }^{27}$ » attaché à produire une sorte de chaleur communautaire autosuffisante. En menant des enquêtes in situ durant plusieurs années, au contact des fidèles, en assistant au culte mais surtout en partageant la vie des convertis, j'ai ainsi pu dépasser cette opposition raison/émotion en tentant de décrire les logiques de socialisation qui prévalent dans ces Églises. Progressivement, la pratique des charismes (prophétie, vision, parler en langues notamment) m'est apparue comme une modalité priante singulière, qui s'apprend et se travaille, comme toute autre pratique rituelle ${ }^{28}$. En participant à des réunions de prière organisées chez des membres des assemblées, j'assistais à de véritables séances d'entrainement aux pratiques charismatiques, destinées à préparer les novices pour la cérémonie de dimanche. Ce type d'observation pouvait alors être mis en relation avec les discours des fidèles, qui s'attachaient lors de nos entretiens à insister sur la dimension spontanée et incontrôlée de leurs propres expressions charismatiques. Les représentations en cours au sein des assemblées pentecôtistes insistent sur l'irruption de l'Esprit Saint lors du culte et sa capacité, selon la formule, à « toucher les cœurs ». La majorité des témoignages de conversion insistent d'ailleurs sur l'imprévisibilité de la conversion, sur l'influence évidente et inattendue de l'Esprit Saint dans la vie des fidèles ${ }^{29}$. La nécessité au sein des assemblées de promouvoir la présence hic et nunc de l'Esprit Saint durant le culte - instrument de légitimation de l'autorité et de confirmation de cette forme de christianisme - permet alors de mieux comprendre la dimension émotionnelle des pratiques charismatiques. L'émotion n'est pas ici un état intérieur qui déclencherait des pratiques priantes et que l'on serait tenté de saisir à partir de notions psychologisantes comme celle de «transe » ou d' «état modifié de conscience ». L'émotion en réalité est un registre de discours particulier, une manière de mettre en scène la dimension imprévisible et incontrôlable du divin, qui mobilise les corps pour se rendre présent en ce monde. Ce sont donc ici les représentations particulières du divin et de ses modalités d'irruption qui conditionnent le recours des fidèles à des modalités priantes mobilisant le registre émotionnel $^{30}$. L'émotion n'est donc pas une cause, c'est une conséquence. Il convient pour le nouveau venu d'apprendre à mobiliser le registre émotionnel lorsqu'il prie avec les autres afin de pouvoir manifester publiquement l'authenticité de sa connexion personnelle avec l'Esprit Saint ; validant du même coup son appartenance à la communauté des élus. On comprend mieux ainsi, grâce à l'enquête ethnographique, à quel point il importe d'être socialisé aux pratiques charismatiques tout en insistant sur la spontanéité des émotions vécues qui, par définition, ne s'apprennent pas. Tout l'enjeu pour ces Églises réside donc dans l'apparent paradoxe d'un « apprentissage de la spontanéité », c'est-à-dire la socialisation à des pratiques de prière qui doivent

25 Laurent Amiotte-SuCHET, «Mettre Dieu dans sa vie. L'apprentissage de la confiance en soi en milieu pentecôtiste français », in Sébastien Fath (dir.), Le protestantisme évangélique, un christianisme de conversion, Turnhout, Brepols, 2004, p. 191-204.

${ }^{26}$ Émile DURKHEIM, Les formes élémentaires de la vie religieuse. Le système totémique en Australie, Paris, PUF, 1968 (1912).

27 Jean-Paul WiLlaime, «Le pentecôtisme : contours et paradoxes d'un protestantisme émotionnel », Archives de sciences sociales des religions 105 (1999), p. 5-28.

${ }^{28}$ Laurent AMIOTTE-SuCHET, «Quand la forme est le fond. Réflexions à partir du parler en langues en milieu pentecôtiste », Revue des Cèdres 36 (2011), p. 67-75.

${ }^{29}$ ID., «Mon corps, ma famille, mon Dieu. Mise en perspective des récits de conversion pentecôtistes », in PierreYves Brandt (dir.), Le récit de soi et la narrativité dans la construction de l'identité religieuse, Paris, Éditions des Archives Contemporaines, 2017, p. 117-135.

${ }^{30}$ Voir ID., «Les médiations de l'immédiateté. L’individualisme communautaire des assemblées pentecôtistes », Théologiques 16/2 (2009), p. 105-121. Voir également Yannick FER, « Genèse des émotions au sein des assemblées de Dieu polynésiennes », Archives de sciences sociales des religions 131-132 (2005), p. 143-163. 
apparaître comme spontanées (non-apprises) pour valider publiquement l'authenticité de la présence de l'Esprit Saint au cœur de l'assemblée.

Je pourrais continuer d'illustrer mon propos à partir d'autres enquêtes, que j'ai menées en milieu catholique, sur les pèlerinages à Lourdes ${ }^{31}$, l'exorcisme contemporain ${ }^{32}$ ou, plus récemment, les couvents et monastères confrontés à leur vieillissement ${ }^{33}$. Sur ces terrains aussi, le recours à la démarche ethnographique (associant observation participante et entretiens compréhensifs) s'est révélé pertinent pour dépasser les représentations ordinaires et montrer les acteurs et les actrices dans toute leur complexité, lorsque chacun et chacune tâtonne au quotidien pour ajuster ses discours et sa pratique. Pas plus hier qu'aujourd'hui, je ne saurais dire ce qu'est un pentecôtiste, un pèlerin, un moine ou une religieuse. Mais ce que l'ethnographie m'a permis de découvrir de leur vie témoigne du travail constant auquel toutes et tous se livrent pour revendiquer leur singularité en ce monde tout en développant des stratégies visant à faire advenir le collectif dont ils pensent être les usufruitiers ${ }^{34}$. C'est ce «religieu[x] en train de se faire ${ }^{35} »$ que l'ethnographie permet de mettre au jour, en évitant à la fois la tentation homogénéisante de l'analyse des communautés et la surinterprétation qui associe pratiques et croyances pour donner une image stable et cohérente des croyants.

La démarche ethnographique est d'abord une méthode que l'on choisit de mettre en œuvre en lien avec une question de recherche. Il ne s'agit pas ici d'être favorable ou défavorable à telle ou telle démarche, en relançant la stérile opposition entre approches qualitatives et approches quantitatives. Il n'y a pas lieu d'établir une hiérarchie entre les différentes méthodologies des sciences sociales. Chacune s'est élaborée et développée pour répondre à certains types de question, chacune correspond à une manière distincte d'envisager l'administration de la preuve.

L'enquête de terrain a néanmoins ses atouts. En proposant au chercheur de s'immerger dans les mondes sociaux qu'il entend étudier, elle lui donne une occasion, bien souvent inédite, de questionner ses préjugés pour se recentrer sur une approche empirique visant d'abord et avant tout à décrire ce que les personnes font, affirment, défendent, revendiquent, sans chercher à tout prix à en déduire ce qu'ils pensent ou croient vraiment. C'est là le principe même d'une démarche inductive, qui donne l'occasion au terrain de faire surgir ce qui se révèlera pertinent pour l'analyse. La réalité ethnographique n'est rien d'autre que ce que le chercheur perçoit depuis la place qu'il a choisi d'occuper. Si cette réalité est toujours singulière, puisqu'elle porte la marque de celui qui observe, elle demeure néanmoins une excellente manière de rendre compte des modalités

31 Laurent AmiotTE-SUCHET, «Les hospitaliers de Lourdes : une communauté événementielle ?», in Ivan Sainsaulieu, Monika Salzbrunn, Laurent Amiotte-Suchet (dir.), Faire communauté en société. La dynamique des appartenances collectives, Rennes, Presses universitaires de Rennes, 2010, p. 75-88.

32 Laurent AmiotTE-SUCHET, «Un ministère de dédiabolisation et de bricolage rituel. Le cas d'un exorciste diocésain », Ethnologie française 46/1 (2016), p. 115-126.

${ }^{33}$ ID., «Les religieuses âgées et leurs soignantes. Hybridité des espaces, des temps et des appartenances dans des institutions de soins », ethnographiques.org 35 (2017) [en ligne] http://www.ethnographiques.org/2017/AmiotteSuchet. Voir aussi Annick AnCHISI, Laurent AMIOTTE-SuCHET, Kevin TOFFEL, «Vieillir au couvent. Stratégies des congrégations et paradoxe des laïcités », Social Compass 63/1 (2016), p. 3-19.

${ }^{34}$ Le fait d'appartenir à une communauté religieuse renvoie autant au passé qu'à l'avenir. Les fidèles se considèrent comme les héritiers d'une longue tradition qu'il leur appartiendra un jour de transmettre. La transmission implique donc toujours trois personnes : celle qui transmet, celle qui reçoit et celle qui devra hériter. Cette approche de la transmission impliquant trois positions a été particulièrement bien analysée par les sociologues du travail et des professions. Voir Dominique JACQUES-JouvenOT, Gilles VIEILlE MARCHISET (dir.), Socio-anthropologie de la transmission, Paris, L'Harmattan, 2012 ; Jean-Claude DAUMAS, « Les dirigeants des entreprises familiales en France, 1970-2010. Recrutement, gouvernance, gestion et performances », Vingtième siècle 114/2 (2012), p. 33-51.

35 Albert PIETTE, La religion de près. L'activité religieuse en train de se faire, Paris, Métailié, 1999. 
d'interaction au sein d'un collectif et, par là même, de la manière dont les individus parviennent à « faire communauté ${ }^{36}$ » en élaborant des frontières sociales et en régulant les expressions publiques (verbales, corporelles) de celles et ceux avec qui ils entendent s'accomplir.

Dans l'étude des faits religieux, l'approche ethnographique a d'abord pour intérêt, de mon point de vue, d'appréhender le religieux comme une activité sociale ordinaire à contextualiser. En évitant ainsi la sacralisation du religieux tout autant que l'essentialisation des croyants, la démarche ethnographique peut se révéler particulièrement pertinente contre les risques de surinterprétation, à condition d'apprendre à ne plus croire à la croyance, et de rester attentif aux actes tout en donnant réellement la parole aux croyants pour qu'ils puissent expliciter ce qu'ils croient sans être immédiatement reléguer à la catégorie réductrice des « croyants ».

Laurent AMIOTTE-SUCHET

${ }^{36}$ I. Sainsaulieu, M. Salzbrunn, L. Amiotte-Suchet (dir.), Faire communauté en société. Dynamique des appartenances collectives, op. cit. 